\title{
LSMO Films with Increased Temperature of MI Transition
}

\author{
M. Španková*, Š. Chromik, E. Dobročka, V. Štrbík, M. Sojková \\ Institute of Electrical Engineering, Slovak Academy of Sciences, Dúbravská cesta 9, 84104 Bratislava, Slovakia
}

\begin{abstract}
Epitaxial $\mathrm{La}_{0.67} \mathrm{Sr}_{0.33} \mathrm{MnO}_{3}$ thin films with a significant increased temperature of metal-insulator transition ( $\sim 450 \mathrm{~K})$ are prepared on single crystal $\mathrm{MgO}(001)$ substrates using different deposition techniques - a dc magnetron sputtering or a pulsed laser deposition. The crystalline perfection of the films is characterized by X-ray diffraction technique (rocking curve measurements and reciprocal space maps). As a consequence of different methods of the film preparation we show various types of the LSMO crystal structure. Our results indicate that all the LSMO layers grown on the $\mathrm{MgO}$ substrate with a lattice misfit of about $8 \%$ are relaxed.
\end{abstract}

DOI: $10.12693 /$ APhysPolA.126.212

PACS: 61.05.cp, 75.47.Lx, 81.15.Cd, 81.15.Fg

\section{Introduction}

Since the discovery of colossal magnetoresistance (CMR) effect in perovskite manganites the magnetic and transport properties of these materials and their relations to microstructure have attracted much interest. Among these materials $\mathrm{La}_{0.67} \mathrm{Sr}_{0.33} \mathrm{MnO}_{3}$ (LSMO) has been intensively studied because of its high Curie temperature $\left(T_{C}\right)$ (higher than room temperature), making this material suitable for uncooled bolometric detectors, sensors, etc. At this temperature the manganites undergo a ferromagnetic-paramagnetic transition and simultaneously, in most cases, a metal-insulator transition - when they reach the resistivity maximum at a temperature $T_{M I}$.

Beside preferred substrates with a perfect matching to the LSMO, significant results on $\mathrm{MgO}$ substrates or $\mathrm{MgO}$ buffer layers have also been obtained with a $T_{C}=365 \mathrm{~K}$ [1], $T_{M I}=416 \mathrm{~K}[2]$ and a $\mathrm{T}_{M I}=382 \mathrm{~K}[3]$, respectively.

LSMO has a perovskite structures with a rhombohedral distortion. In the pseudocubic description the lattice parameter and the unit cell angle are $0.3876 \mathrm{~nm}$ and $90.26^{\circ}$, respectively. The $\mathrm{MgO}$ substrate has the rocksalt cubic structure with a lattice parameter of $0.421 \mathrm{~nm}$. The lattice mismatch between the LSMO film and the $\mathrm{MgO}$ substrate is $8 \%$.

\section{Experimental details}

The LSMO films were deposited using two different deposition techniques, on axis dc magnetron sputtering or pulsed laser deposition (PLD) onto a one-side polished $\mathrm{MgO}(001)$ substrate. In case of sputtering the deposition was performed in an $\mathrm{Ar}(80 \%)+\mathrm{O}_{2}(20 \%)$ atmosphere at a total pressure of $65 \mathrm{~Pa}$. During the deposition, the substrate was heated to $800{ }^{\circ} \mathrm{C}$. In order to increase the oxygen content, the films were subsequently in-situ annealed

*corresponding author; e-mail: marianna.spankova@savba.sk in $\mathrm{O}_{2}\left(10^{4} \mathrm{~Pa}\right)$ at $800{ }^{\circ} \mathrm{C}$ for an hour. In case of PLD a $\mathrm{KrF}$ excimer laser operating at $248 \mathrm{~nm}$ with a pulse width of $20 \mathrm{~ns}$, a repetition rate of $10 \mathrm{~Hz}$ and an energy density of $2 \mathrm{~W} / \mathrm{cm}^{2}$ was used to grow the LSMO films. A temperature of substrate holder of $850{ }^{\circ} \mathrm{C}$ and oxygen pressure of $53 \mathrm{~Pa}$ were set during the deposition. After the deposition the films were cooled down $20{ }^{\circ} \mathrm{C} / \mathrm{min}$ in $\mathrm{O}_{2}\left(4 \times 10^{4} \mathrm{~Pa}\right)$.

The thickness of the films was about $50 \mathrm{~nm}$, the growth rate of the sputtered and the laser ablated LSMO were $1.2 \mathrm{~nm} / \mathrm{min}$ and $6.5 \mathrm{~nm} / \mathrm{min}$, respectively.

The temperature dependence of the resistance was measured using a standard four-probe technique in a temperature range of $4-480 \mathrm{~K}$.

XRD analyses $(\theta-2 \theta$ scans, rocking curves, reciprocal space maps) were carried out using Bruker D8 DISCOVER diffractometer equipped with X-ray tube with rotating $\mathrm{Cu}$ anode operating at $12 \mathrm{~kW}$. All measurements were performed in parallel beam geometry with medium resolution set-up with parabolic Goebel mirror in the primary beam but without monochromator.

\section{Results and discussion}

The resistance vs. temperature $(R-T)$ dependences of the LSMO films prepared by both dc magnetron sputtering and PLD methods exhibit usually a broader resistance peak with maximal values in temperature range above $400 \mathrm{~K}$ (Fig. 1). The temperature of the maximal resistance, denoted as $T_{M I}$, (temperature of metalinsulator transition, Fig. 1, inset) is significantly higher than the LSMO bulk value $(370 \mathrm{~K})$ and corresponds to the onset temperature of the ferromagnetic transition in the LSMO films. The increased values of the $T_{M I}$ and temperature of the ferromagnetic transition are ascribed to an increased strain in the films $[2,4]$.

XRD measurements carried out in $\theta-2 \theta$ configuration indicate that both types of the LSMO films exhibit only $00 l$ orientation. The degree of the preferred orientation of the LSMO films, perpendicular to the $\mathrm{MgO}$ substrate surface, was deduced from the full width at half maximum (FWHM) of the rocking curve. The FWHM value 


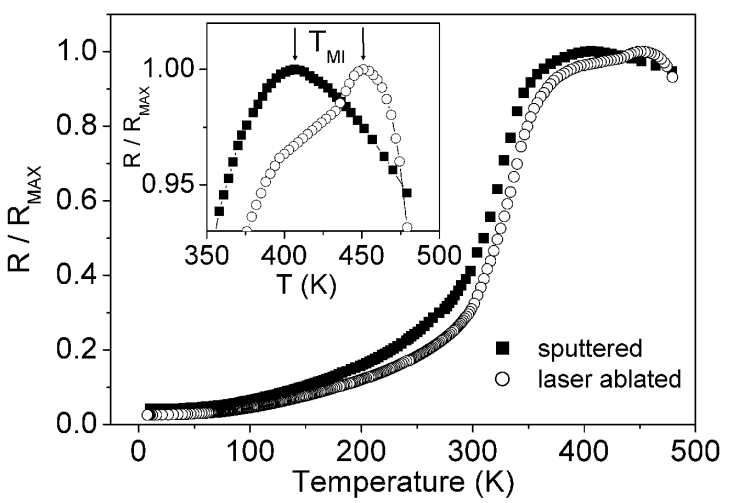

Fig. 1. R-T dependences of two LSMO films with enhanced values of $T_{M I}$ prepared by different methods. The top of the resistance peak for both films is shown in the inset.
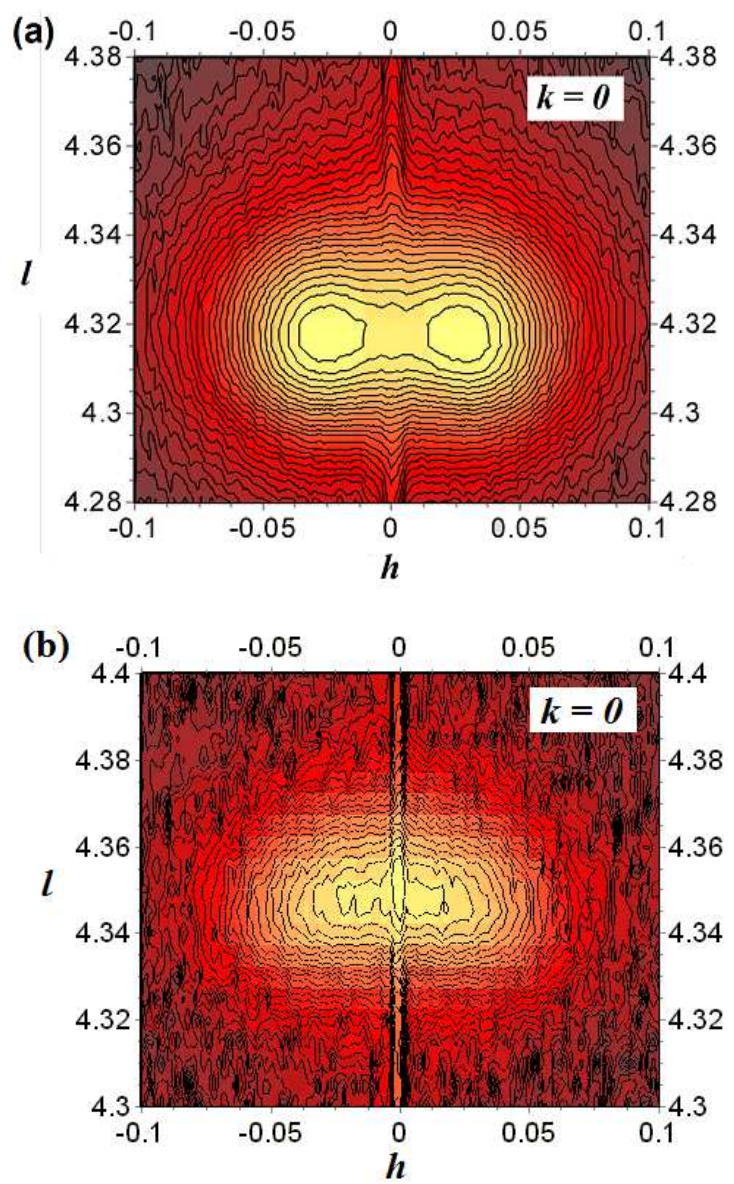

Fig. 2. XRD analyses (reciprocal space maps around $004_{C}$ diffraction) showing the difference in the growth of the sputtered (a) and laser deposited (b) LSMO films. measured on the 004 LSMO peak reached systematically $1-1.3^{\circ}$. However, we have revealed different shapes of the rocking curves of the LSMO - in case of the sputtered LSMO film two distinct maxima of the rocking curve, while in case of the laser ablated LSMO film a simple shape of the rocking curve with one maximum were observed. To explain the origin of the rocking curve splitting, the reciprocal space maps were recorded around the symmetric 004 and asymmetric 204 Bragg diffractions (Fig. 2a and 2b). The LSMO films prepared by sputtering exhibited splitted rocking curves in the direction parallel to the sample surface, indicating a distorted orthorombic LSMO unit cell with [110] out-of-plane orientation and [1-10] and [001] in-plane orientations. The out-of plane lattice parameter $a_{C}^{\perp}$ of the sputtered LSMO, found by X-ray diffraction in symmetric configuration, is $0.3899 \mathrm{~nm}$. The in-plane parameter $\mathrm{a}_{C}^{\|}$, deduced from the asymmetric diffraction, reaches a value of $0.3865 \mathrm{~nm}$. On the other hand, no splitting of the rocking curves was observed in case of laser ablated films. These films possess a pseudocubic structure with $a_{C}^{\perp}$ parameter of $0.3871 \mathrm{~nm}$ and $\mathrm{a}_{C}^{\|}$parameter of $0.3844 \mathrm{~nm}$.

XRD analyses have revealed that both types of the LSMO films grown on the $\mathrm{MgO}$ are relaxed with respect to the substrate. The LSMO lattice parameters are different from the LSMO bulk value, indicating a presence of some strains in the LSMO films. These strains are created during the film growth and are brought into by the adjusting of the deposition conditions of the films.

\section{Conclusions}

We prepared epitaxial LSMO films using different deposition techniques. According to XRD measurements the crystal structures of the sputtered and the laser ablated LSMO films are orthorhombic and pseudocubic, respectively. The unit cell parameters calculated from the positions of the diffraction maxima in the reciprocal space maps indicate an existence of compressive stress in the LSMO layers. Regardless of the deposition method, our LSMO films exhibited significantly higher $T_{M I}$ values than the bulk value.

\section{Acknowledgments}

This work was supported by the Slovak Research and Development Agency, APVV-0494-11, APVV LPP-007807, Slovak Grant Agency for Science, VEGA 2/0164/11, and the project CENTE II, R\&D Operational Program funded by the ERDF, ITMS code 26240120019.

\section{References}

[1] S.Y. Yang, W.L Kuang, C.H. Ho, W.S. Tse, M.T. Lin, S.F. Lee, Y. Liou, Y.D. Yao, J. Magn. Magn. Mater. 226-230, 690 (2001).

[2] Š. Chromik, V. Štrbík, E. Dobročka, A. Dujavová, M. Reiffers, J. Liday, M. Španková, Appl. Surf. Sci. 269, 98 (2013).

[3] I. Khartsev, J.-H. Kim, A.M. Grishin, J. Cryst. Gr. 284, 1 (2005).

[4] A. Sadoc, B. Mercey, Ch. Simon, D. Grebille, W. Prellier, M.-B. Lepetit, Phys. Rev. Lett. 104, 046804 (2010). 